\title{
A New Composite Algorithm for Identifying the Stars in the Star Tracker
}

\author{
Ali Reza Toloei \\ Department of Aerospace \\ Shahid Beheshti University \\ Tehran, Iran
}

\author{
Morteza Shayan Arani \\ Department of Aerospace \\ Shahid Beheshti University \\ Tehran, Iran
}

\author{
Manouchehr Abaszadeh \\ Department of Aerospace \\ Shahid Beheshti University \\ Tehran, Iran
}

\begin{abstract}
Identifying the stars of field of view(FOV) is a difficult stage in determining the attitude through star tracker. Stars identification algorithms identify the stars of FOV, creating patterns from the stars and comparing them with the information saved in the memory. This research presents a new algorithm composed of pyramid, liebe and planer triangle algorithms. It has at least 4 stars as the stars of FOV and forms a triangle pattern. This algorithm is simulated with 16000 stars in MATLAB. Its accuracy, needed memory and searching time are studied and compared with those of pyramid, Liebe and triangular methods. Two errors, centroiding and in fake stars, are considered in the simulation for more approaching towards real status. According to the obtained results, the new algorithm can well identify the FOV among 140 stars in which only four stars are real and the rests are false. Its searching time is $8 \%$ lower, comparing to pyramid method, despite their similar accuracies. It compares the areas and polar moments of the four triangles with the information saved in the memory to meet a unique response. Besides its robustness and proper performance, it includes the false stars as well.
\end{abstract}

\section{General Terms}

Star Identification Algorithms, Search Ranging Method, Star Pattern

\section{Keywords}

Star Tracker; Attitude Determination, Pyramid Algorithm, Star Catalogue, Triangle Algorithm, Liebe Algorithm, Centroiding Error.

\section{INTRODUCTION}

Accurate attitude determination is becoming more and more important for the success of space missions, as the requirements on spacecraft attitude and angular rate control become more stringent. Sometimes, the least error can cause severe aftermath. Different devices have been designed and constructed in this regard each of which identifies the situation with its own criteria. Magnetometers sensor, Gyroscope, Sun sensor and Star tracker are of the navigation devices which are discussed briefly in the following.

\footnotetext{
Magnetometers determination attitude identify the spacecraft condition through measuring the size and orientation of magnetic field of the ground. As the magnetic field is not well known for us, these devices have no significant accuracies[1]. Gyroscope determination attitude identify the spacecraft condition with measuring the torques applied on the axis. In order to achieve high accuracy, a particular technology should be used in its construction which causes the higher cost of this navigation aid device[1].
}

Sun sensor determines the spacecraft attitude with respecting to the situation of sun. As this device should always be exposed to the sun radiation, it has shorter life comparing to other sensors[1].

Star tracker is a sensor widely considered in the recent years due to its high accuracy. This device has been used in the airplanes in 40s, contemporary with the Second World War, for the first time. Then, in the 50s, it has been applied as an aid device along with gyroscope to correct the gyroscope errors, created and increased in time. Since then, a tracker similar to the modern ones has been used in the lunar project for the first time with the aim of stability in the earth orbit. New generation of star trackers do not need the primary information of the spacecraft condition and can calculate it in the real time with the accuracy of $1 \mathrm{arc} / \mathrm{sec}$. The sensors are presented in Table 1.

Table 1 . Comparing the sensors

\begin{tabular}{ccc}
\hline Sensors & General specifications & $\begin{array}{c}\text { Accuracy } \\
\text { range }\end{array}$ \\
\hline $\begin{array}{c}\text { Sun sensor } \\
\text { Magnetic } \\
\text { sensor }\end{array}$ & $\begin{array}{c}\text { Easy, cheap } \\
\text { Costly, low accurate, used } \\
\text { in lower heights } \\
\text { Dyroscope }\end{array}$ & $\begin{array}{c}\text { Difficult calibration, } \\
\text { increasing error with time }\end{array}$ \\
Star tracker & $\begin{array}{c}\text { Heavy, complex, accurate, } \\
\text { expensive }\end{array}$ & $0.3 \mathrm{deg}$. \\
\hline 2. ATTITUDE DETERMINATION BY \\
STAR TRACKER
\end{tabular}

The star trackers determine the spacecraft attitude by taking image of the sky and comparing the stars existed in their FOV with the information saved in the stars catalogue. Centroiding algorithm, Stars identifying algorithm and Attitude estimating algorithm construct the software structure of star trackers.

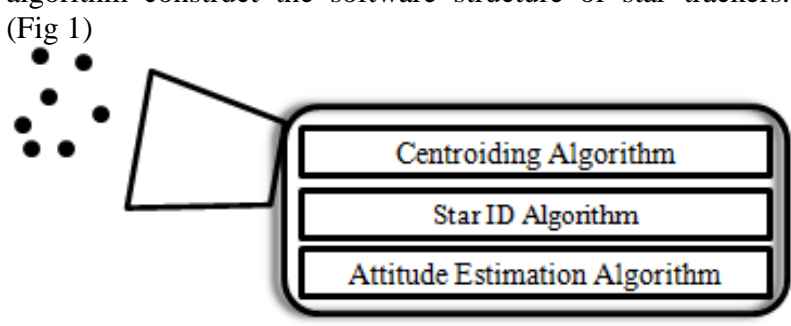

Fig 1. Software structure of star tracker

Centroiding algorithms are used in the star trackers to locate precisely each star in the centers of detector pixels. After these algorithms, how to identify the stars existed in the FOV is one of the difficult and effective steps in the performance of 
star trackers which is performed by stars identification algorithms. In this stage, the body vectors are converted to the vectors of inertial system in the images. Then the spacecraft attitude is determined by using attitude estimation algorithms(Fig 2). These days, in order to increase the rate of searching, the algorithms have been presented with the aim of faster and easier searching in the high volume saved data. They significantly affect the rate of star trackers.

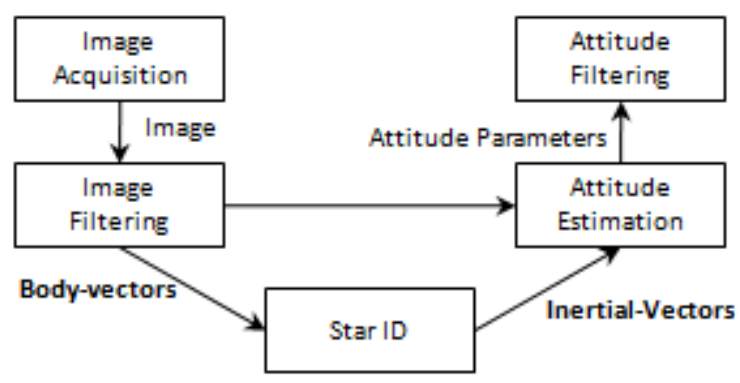

Fig 2. Typical spacecraft autonomous attitude determination process[6]

\section{STARS IDENTIFICATION ALGORITHMS}

In the early 70th it was started to attempt for accessing the algorithms for identification the stars of FOV. At the time, angular algorithm was presented by Gotlieb and then in 1986 triangle algorithm by Grouse.The latter has better performance comparing to the former. In 1991 Anderson and Junkins searched for an algorithm to identify the stars and be able to act in an star tracker located in the lower orbits and confront the noises in such heights. They considered new parameters in their algorithm for feature lists with high data volume. Next year (1992), Renken used the distances between the pixels of images presenting them as the algorithm for stars identification. This algorithm could well resist against the noises of optical part of the tracker as well as the error of stars catalogue. After then, in the same year, Liebe presented an algorithm in which the saved data volume had been reduced significantly. This fact accelerated the speed of algorithm; however, his algorithm had improper capability in confronting the revealed errors. Baldini (1993) presented the measuring of 5 stars among 5 consecutive stars in the FOV. Mortari (2004) suggested the pyramid algorithm and $\mathrm{K}$ vector searching method. This is still the most effective stars identification algorithm and used in different projects. Zhang (2007) presented an algorithm based on the Liebe excluding the applying of polar coordinates which caused the distinction of his algorithm among the existed ones. Tichy (2011) proposed an algorithm based on internal multiplying of two 3demensional vectors showing the location of stars. He needed two stars in his algorithm for starting the stars identification.

\subsection{Typical star identification process}

Stars identification algorithms are classified into two groups concerning the situation in which the spacecraft is located. The first group is when the spacecraft has been recently launched to the border and no information about its attitude is available. This status is called Lost In Space (LIS). The algorithms used in this mode need more time and memory volumes for identifying the stars of FOV. The other status is when the attitude of spacecraft has been distinguished several earlier moments and its primary information is available. This status is called recursive or tracking mode. Stars identification and attitude determination are easier and faster in this mode. In the lost in space mode, the star identification algorithms need more stars in the FOV, comparing to the tracking mode through which the attitude can be defined even with only one star. Liebe algorithm, pyramid algorithm and the suggested algorithm are of the lost in space ones in this research. General stages of stars identification has been presented in an algorithm as follows:

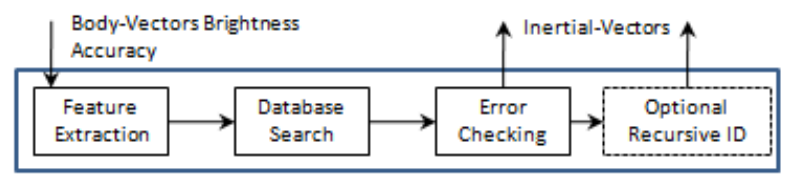

Fig 3. Typical star identification process[6]

According to the Fig 3, speed and accuracy of stars identification algorithms depend on the saved data volume, created feature lists numbers and the way of their organization as well as the searching procedures of these high volumes of saved information. Therefore, the speed of stars identification can increase by decreasing the saved data volume and its appropriate alignment. In 1970, it was attempted to present the algorithms for lost in space status. Since then, considerable researches have been conducted on the reducing of saved information and organizing it in these algorithms. Moreover, many algorithms have been suggested for faster searching among the data, the most successful of which is kvector searching algorithm. This algorithm is the proper substitute for binary search methods of the data. It does not depend on the saved data volume in addition to have the speed of 10-50 times that of binary methods.

\subsection{Planer triangular Stars-ID algorithm}

The firs methods for identifying the stars have been presented in 1978 by Gottlieb and named as the method of separating angle between two stars of the FOV[4]. Regarding the high probability of the existence of several star pares with equal separation angles, very often unique response was hardly achieved at the end of algorithm. Therefore, triangular algorithm has been presented by Groth in 1986 to create more parameters for comparing the stars of FOV with star catalogue. In this algorithm first, the areas that should be created by each three stars are calculated and saved in the feature list. Then, the polar moment of these triangles are computed (Fig 4) and put in the feature list. By the way, there will be very few triangles with equal areas and polar torques. It should be mentioned that in the triangular algorithm there should be at least three stars in the FOV. In contrast, only two stars are enough for identifying through angular method. It should be noted that the angular method has no high reliability and it is most probable to meet unreal response there[7].

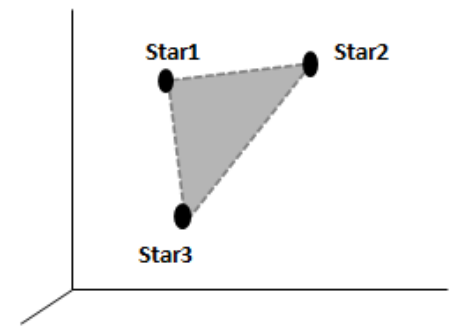

Fig 4. Planer Triangle method pattern 


\subsection{Liebe Stars-ID algorithm}

Liebe used three stars in the FOV in his algorithm suggested in 1995. He selected one star as the central one and then two others in such a way to be the nearest to the central star. Liebe calculated two angles between the central star and the second and third stars. Then, he proposed two parameters for comparing them with star catalogue, Fig 5. Besides the mentioned two angles, Liebe computed an internal angle between the second and third stars with the centrality of central star. In this method, each star is selected as the central one and the angle between two others is obtained and saved in the feature lists[1].

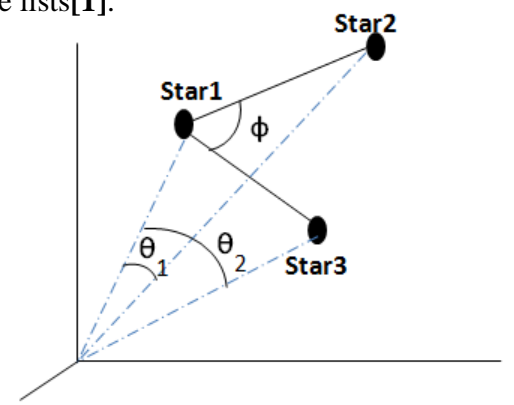

Fig 5. Liebe Star ID algorithm pattern

In Liebe method, as only near stars are selected as criteria, creating pattern is more limited, comparing to the procedures which use all stars. Besides, this limitation causes the automatic decrease of the saved data in the star tracker resulting in the faster searching application. Liebe algorithm flow diagram has been presented in Fig 6 .

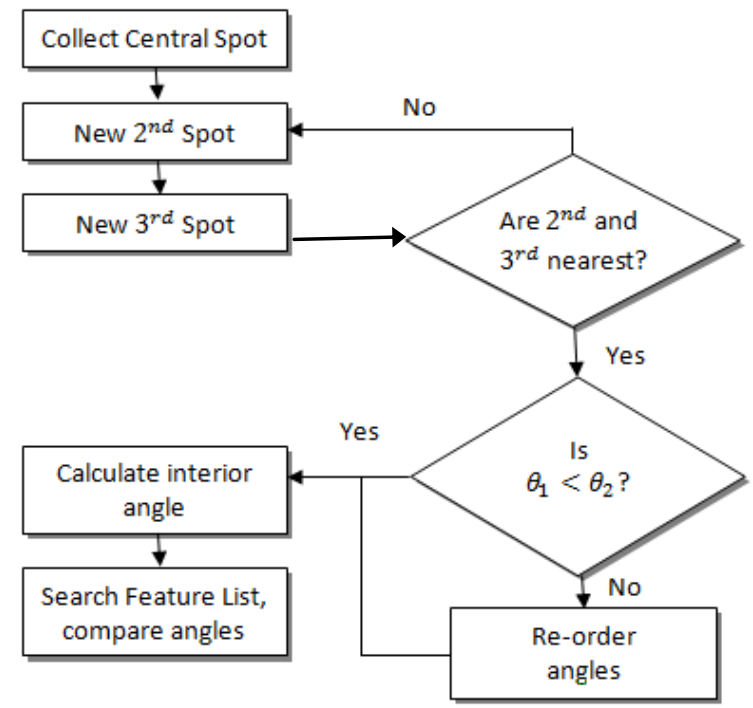

Fig 6. Flow diagram of Liebe Star ID algorithm

\subsection{Pyramid Stars-ID algorithm}

Pyramid algorithm is of the algorithms tested in the space missions presenting appropriate performances. This algorithm has been suggested by Mortari (2004). Pyramid algorithm needs at least 4 stars in its field of view. In this algorithm, first, a triangle is formed by three stars and named primary triangle. Then, the forth star is selected out of the stars remained in the FOV as the reference star which forms the vertex of considered pyramid, Fig 7.

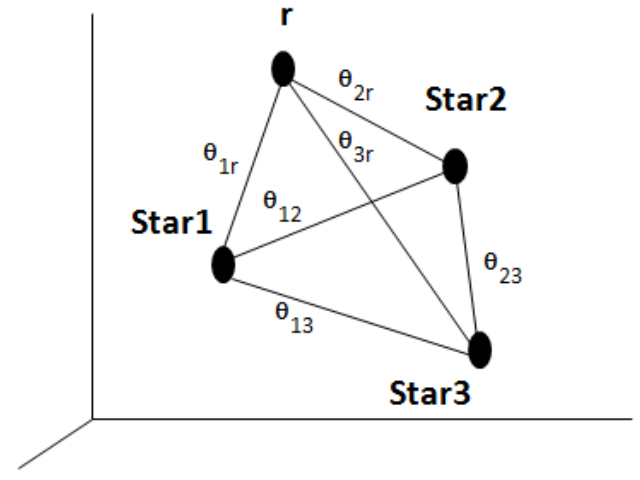

Fig 7. Pyramid Star ID algorithm pattern

Now, the unique response is searched among the feature lists by comparing the angles between the reference star and each vertices of the primary triangle with the saved data. In case of not achieving the unique response, another star of the FOV is selected as the reference and the searching and comparing functions are performed again. The identification parameters in the pyramid algorithm are three angles in the primary triangle and three angles between the reference star and the stars of primary angle vertices. The high numbers of parameters in this algorithm result in its high reliability. In the pyramid algorithm, a more advanced method, called kvector searching[2], is used instead of binary searching method that minimizes search time. Besides, the smart method is used to create the triangles formed by composing three different stars which is the optimum method in this regard[3].

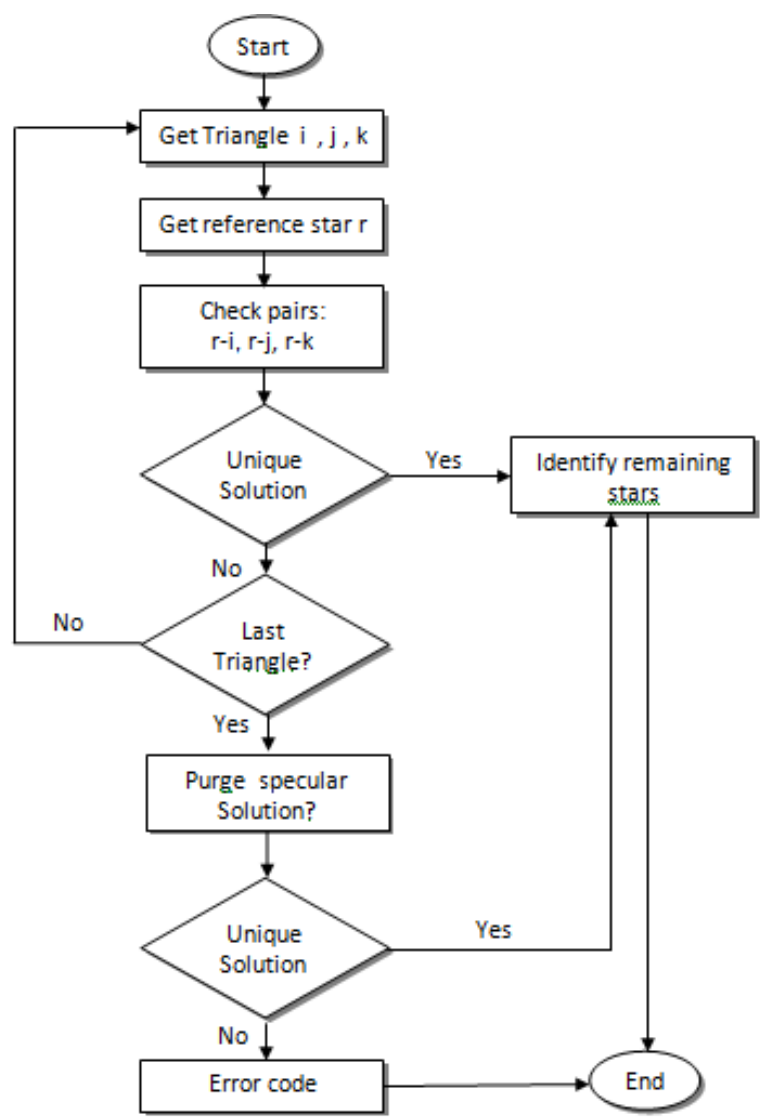

Fig 8. Flow diagram of pyramid star ID 


\subsection{The novel Stars-ID algorithm}

The algorithm proposed in this research has been deduced from pyramid and planer triangular methods. In this algorithm a matrix has been considered with 5 columns as feature list. The first three columns are the numbers of stars which form the whole triangles existed in the star catalogue. The triangles have been selected optimally and without repeating according to the smart method presented by Mortari. The third and forth columns are representatives of areas and polar torques of the triangles, respectively. In order to start the searching activities, firstly, the base triangle should be formed, like pyramid method, and then its exact existing should be ensured. That is three stars of FOV are selected at first; and then the area and torque of the obtained triangle are calculated. If a triangle is formed with the area and torque equal to the feature lists, the second step is started; otherwise, another three stars are selected from FOV. It should be mentioned that the $\mathrm{K}$ - vector method has been used in this algorithm for minimize searching time. K- vector method has been used by Mortari in the pyramid algorithm. The advantage of this method is its independence on the data size. After obtaining the basic triangle, a star is selected out of others in the FOV to form a pyramid with the basic triangle in order to verify the response which is the three stars of the basic triangle. Now, the areas and polar torques of the three triangles are calculated and searched which are the vertices of pyramid. If the unique response is obtained, then four stars are identified; otherwise, another star is selected in the FOV as the vertex star of the pyramid. If all stars of the FOV are controlled for reference and no unique and proper response is obtained, then another three stars are considered as constitutive stars of basic triangle and the activities are repeated up to the identification of four stars. The areas and polar torques of the triangles are obtained according to the below relations with respecting to the coordinate vectors.

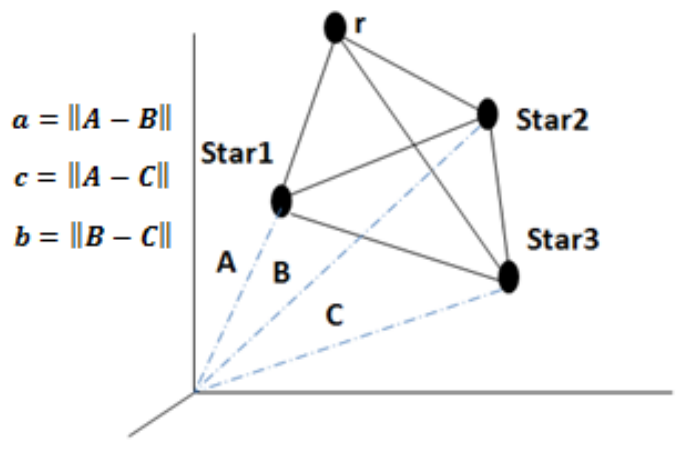

Fig 9. Novel star ID pattern

$$
\begin{gathered}
S=\frac{1}{2}(a+b+c)(\text { Eq.1) } \\
I=\frac{A\left(a^{2}+b^{2}+c^{2}\right)}{36}(\text { Eq.2 })
\end{gathered}
$$

The flow diagram of the suggested algorithm is presented in below(Fig 10):

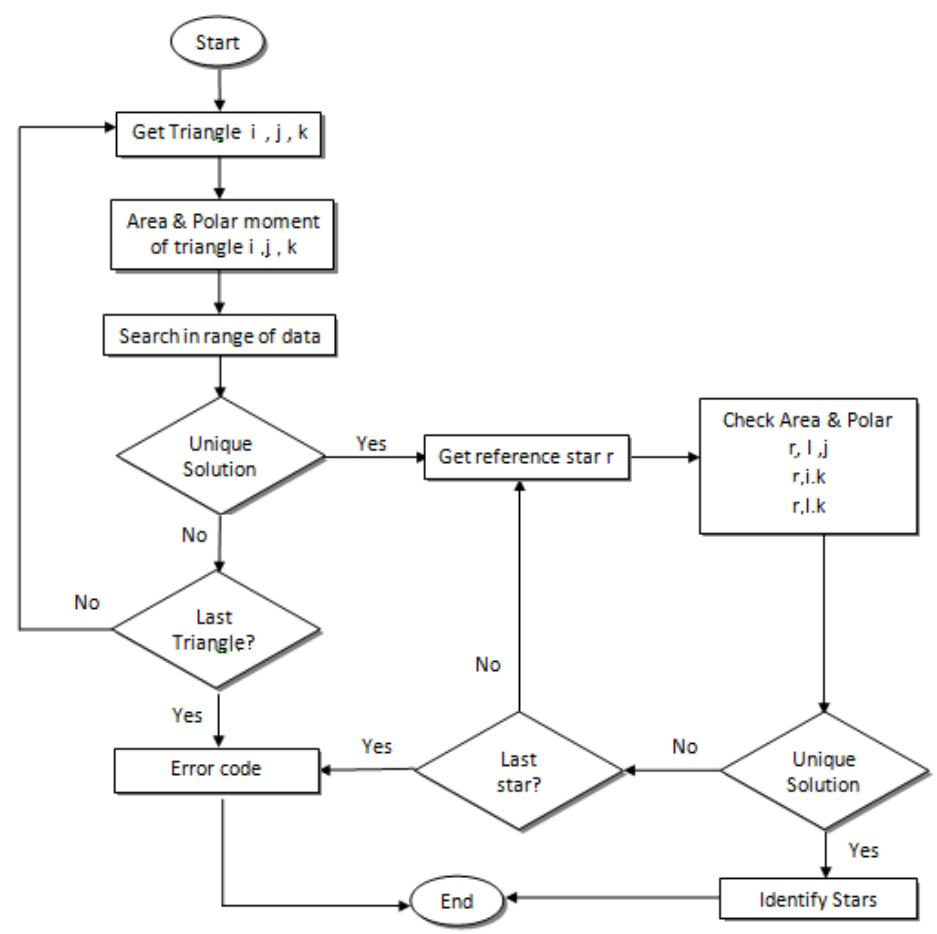

Fig 10. Flow diagram of novel star ID algorithm

\section{SIMULATING THE SUGGESTED ALGORITHM}

The stars of the catalogue and FOV are simulated for assessing the accuracy and efficiency of the suggested algorithm. The simulated star catalogue is a hypothetical catalogue, the coordinates of which have been randomly assigned. The star catalogue with 2000 stars have been presented in the below figure:

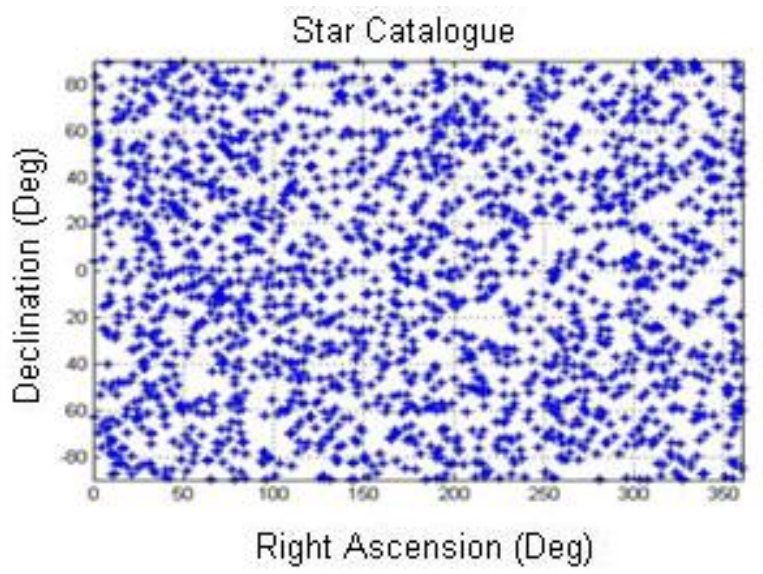

Fig 11. Simulated star catalogue

Two types of errors occur more frequently in the star tracker comparing to other arenas and make difficulties in the identification of stars of the FOV. These errors are considered in the simulation for approaching the simulating condition to the reality. One type of the errors occurs due to the presence of another planet or satellite in the FOV causing the formation of a false star in the FOV of star tracker; while, such star does 
not exist in the star catalogue or feature lists. Such errors can even happen because of the light reflection from the satellite body on which the star tracker has been installed. In Fig 12 false stares are shown with black color and those existed in the star tracker with green color. The red rectangular is the FOV of the star tracker camera. The stars of the FOV have been reduced for more clarity.

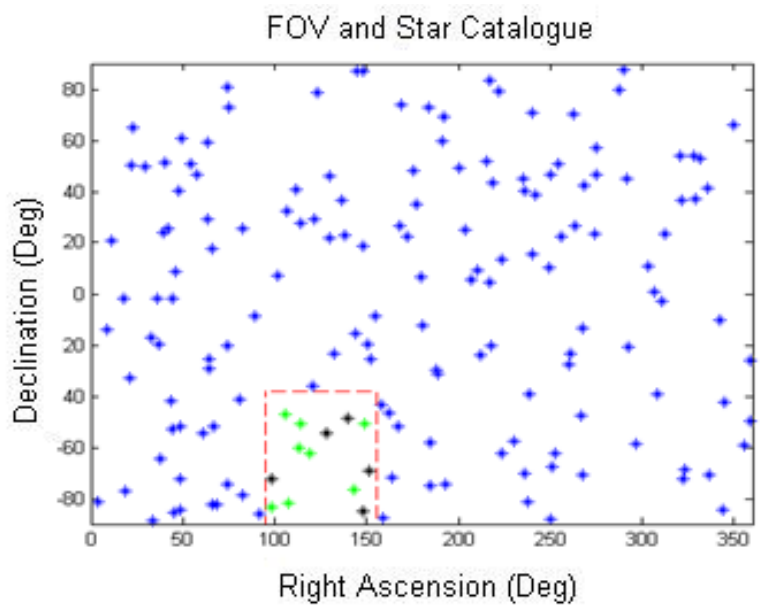

Fig 12. Real stars and Fake stars in FOV

Another type of error is happened because of not correctly matching the stars in the pixel centers of the camera. Such error makes deviation in the locations of stars of FOV relative to the stars saved in the catalogue. Fig 13 shows the deviation of stars locations in the star tracker caused by the error of central algorithm. Green points are the real situations of stars and the red points are the ones observed by stars tracker, different from the real situations due to the error of central algorithm.

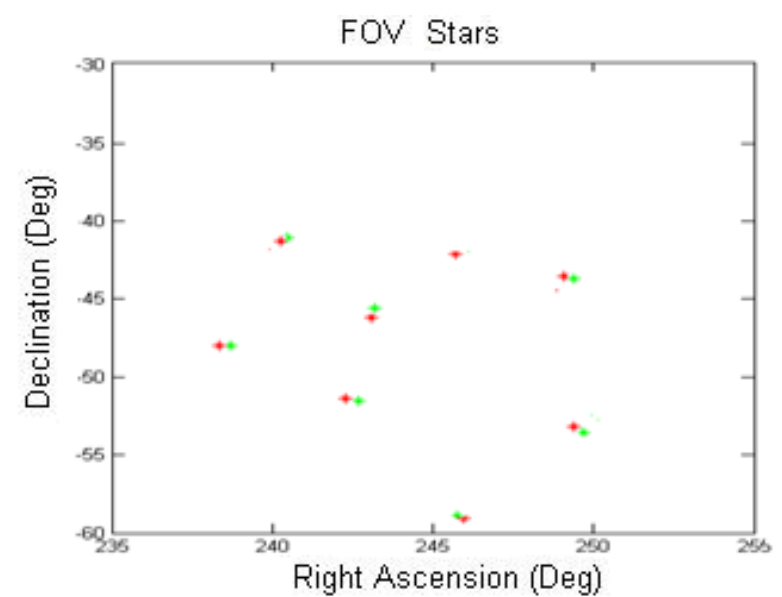

Fig 13. Centroiding error in star tracker

According to the results obtained by simulation in MATLAB software, the suggested algorithm has the relatively high volume of needed memory, comparing to Liebe method. It means that it needs a memory of 12-14 times that of Liebe for saving the information. Concerning the triangle and pyramid methods, all three algorithms need equal memories, despite considering 6 parameters in the feature list in the pyramid method (3 internal angles and 3 angular distances for each triangles).

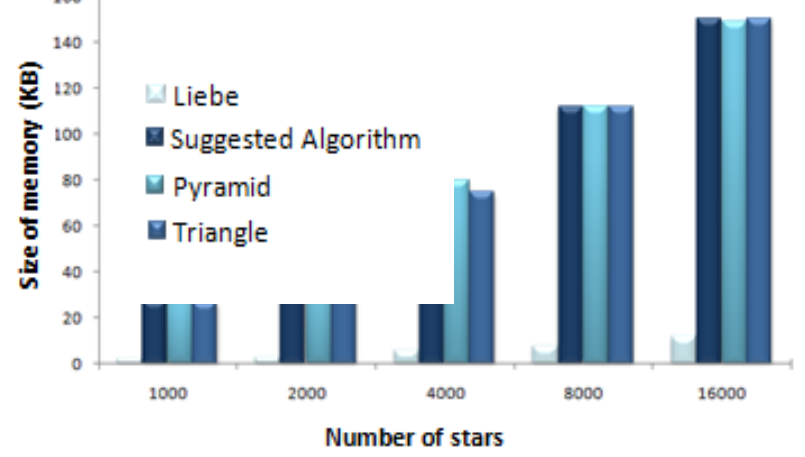

Fig 14. Comparing the algorithms based on the needed memory

The response accuracy and research time are two factors effective in evaluating the performance of star identification algorithm.

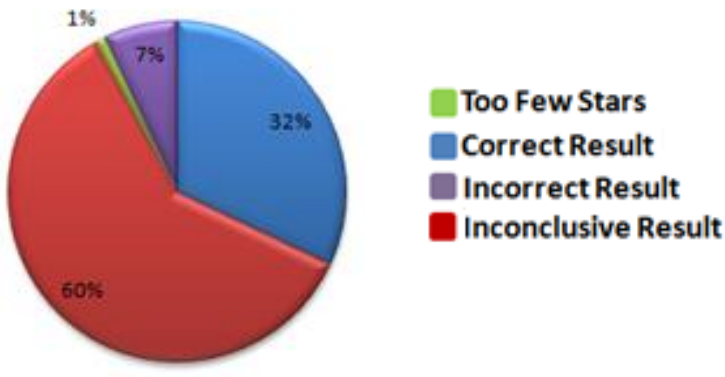

Fig 15. Liebe Star ID

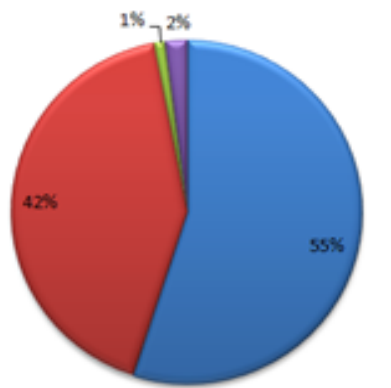

Too Few Stars

Correct Result

Incorrect Result

Inconclusive Result

Fig 16. Planer Triangle Star ID

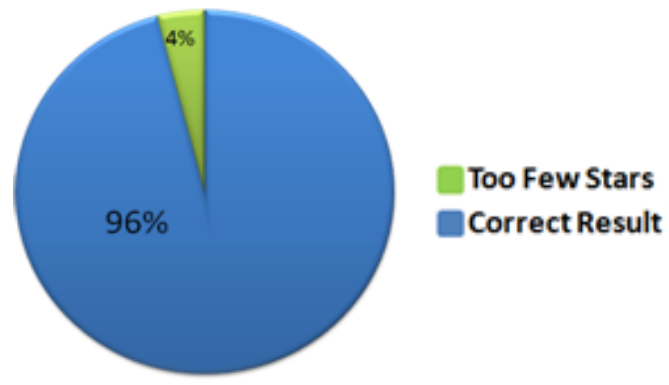

Fig 17. Pyramid and novel star ID algorithm results with fake stars

In the Liebe method the volumes of feature lists are reduced by creating the pattern with close stars; therefore, this method has higher speed comparing to others. The suggested method and pyramid method have the same accuracies; however, in 
the latter the time for achieving response is lower $8-10 \%$ in comparison to the former, Fig 18.

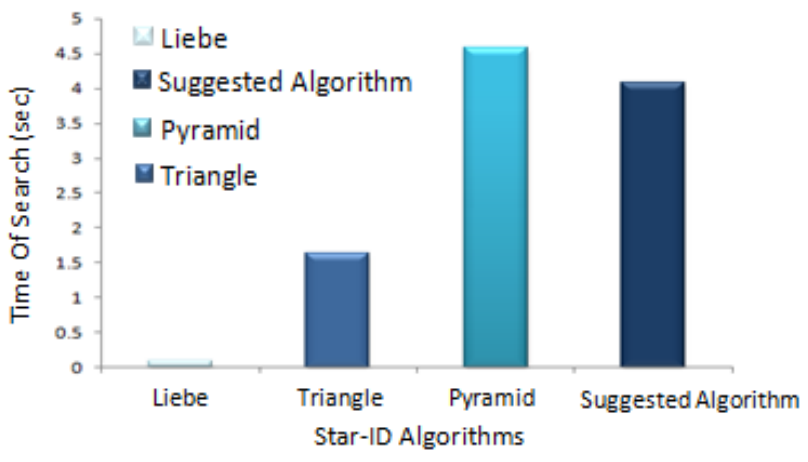

Fig 18. Comparing the algorithms based on the time of search

\section{CONCLUSION}

This research presents an algorithm which needs at least four stars in its FOV for identifying the stars. It can determine the spacecraft attitude in the lost phases of space as well. This algorithm needs more memory comparing to Liebe and angle algorithms; however, has much higher capability like pyramid method. When only 4 stars out of 140 stars of the FOV are real, this algorithm can successfully identify the stars; this is an appropriate statistics for an algorithm. Meanwhile, the research time is $8 \%$ lower in the proposed algorithm due to its fewer parameters, comparing to the traditional pyramid method.

\section{REFERENCES}

[1] Liebe, C. C. Pattern recognition of star constellations for spacecraft applications. IEEE Aeronaut. Electron. Syst. Mag. 1992, 10, 2-12.

[2] Mortari,D. K-vector range searching techniques. Adv. Astronaut. Sci. 2000, 105, 449- 464.

[3] Mortari, D. Samaan, M. A. Bruccoleri, C. The pyramid star identification technique. Navigation 2004, 51, 171 183 .

[4] Steven Bratt. Analysis of Star Identification Algorithms due to Uncomensated Spatial Distortion. Master's thesis, 2013.

[5] Kara M .Huffman. Designing Star Trackers to Meet Micro-satellite Requirements. Master of science in Aeronautics and Astronautics, 2006.

[6] Benjamin Barett Spratlin. Star-ND (Multi-Dimensional Star-Identification).Texas A\&M University, 2011.

[7] Craig. L. Cole, John L. Crassidis. Fast Star Pattern Recognition Using Planar Triangles. AIAA, 2005.

[8] Malak A. Samaan .Toward Faster And More Accurate Star Sensors Using Recursive Centroiding and Star Identification.Texas A\&M University, Doctor of Philosophy, 2003.

[9] Meng Na, Peifa Jia. A survey of All-Sky Autonomous Star Identification Algorithms. IEEE, 2009.

[10] Malak A. Samaan, Daniele Mortari. Recursive Mode Star Identification Algorithms. IEEE, 2005. 